\title{
Uniform distribution of the Steinitz invariants of quadratic and cubic extensions
}

\author{
Anthony C. Kable and David J. Wright
}

\begin{abstract}
It is shown that the Steinitz invariants of the cubic extensions of a number field are uniformly distributed in the class group when the cubic extensions are ordered by the ideal norm of their relative discriminants. This remains true even if the extensions are restricted by specifying their splitting type at a finite number of places. The same statement is also proved for quadratic extensions.
\end{abstract}

\section{Introduction}

Algebraic number theory began with a focus on the arithmetic of number fields viewed as extensions of the rational numbers. As the subject developed, its internal logic forced a change in perspective: any number field $k$ might serve as the domain of rationality and extensions of $k$ were to be studied as such. As the ring of integers, $\mathcal{O}_{k}$, of $k$ is not generally a PID, new phenomena appear in this relative setting. For example, there might be finitely-generated torsion-free $\mathcal{O}_{k}$-modules that are not free. There is an invariant of such $\mathcal{O}_{k}$-modules, the Steinitz invariant, which takes its values in the ideal class group, $\mathrm{Cl}(k)$, of $k$ and quantifies this phenomenon. The rings of integers of finite extensions of $k$ are an abundant and important source of finitely-generated torsion-free $\mathcal{O}_{k}$-modules and it is natural to inquire about the properties of their Steinitz invariants. There is substantial and growing literature that studies this question by algebraic methods [Art65, Car98, Fro60, God02, GS03, KP00, Sov02]. Here we use analytic methods to establish an equidistribution result for the Steinitz invariant of the ring of integers of a quadratic or cubic extension of a fixed number field. We may state a simplified version of our main result, Corollary 5.6, as follows.

Theorem 1.1. Let $k$ be a number field of class number $h$ and fix $\mathcal{e} \in \mathrm{Cl}(k)$. If $M$ is a finitelygenerated torsion-free $\mathcal{O}_{k}$-module then let $S(M)$ denote its Steinitz invariant. If $K$ is a finite extension of $k$ then denote by $\Delta_{K / k}$ the relative discriminant of $K$ over $k$ and by $\mathcal{N}\left(\Delta_{K / k}\right)$ the absolute ideal norm of $\Delta_{K / k}$. For $d=2$ or 3 we have

$$
\lim _{B \rightarrow \infty} \frac{\left|\left\{K \mid[K: k]=d, \mathcal{N}\left(\Delta_{K / k}\right) \leqslant B, S\left(\mathcal{O}_{K}\right)=\mathcal{C}\right\}\right|}{\left|\left\{K \mid[K: k]=d, \mathcal{N}\left(\Delta_{K / k}\right) \leqslant B\right\}\right|}=\frac{1}{h},
$$

where the vertical bars stand for cardinality.

As mentioned in the abstract, the full version of this result allows the extension $K$ to be restricted by specifying its completion, up to isomorphism, at each of finitely-many places of $k$. Also, for $d=3$, the contribution to the numerator and denominator from cyclic cubic extensions is negligible and so they may be excluded. The conclusion remains the same.

Received 6 April 2004, accepted in final form 27 April 2005.

2000 Mathematics Subject Classification 11R45 (primary), 11R11, 11R16, 11S90, 11M41 (secondary).

Keywords: Steinitz invariant, cubic extension, equidistribution, Shintani zeta function.

The first-named author was partially supported by NSF grant DMS-0244741.

This journal is (c) Foundation Compositio Mathematica 2006. 


\section{UNIFORM DISTRIBUTION}

In [Fro60], Fröhlich proved that every class in $\mathrm{Cl}(k)$ is the Steinitz invariant of $\mathcal{O}_{K}$ for some quadratic extension $K$ of $k$. For $d=2$, our result is a refinement of Fröhlich's. For $d=3$, it has as a corollary that every class in $\mathrm{Cl}(k)$ may be realized as the Steinitz invariant of a non-normal cubic extension of $k$.

We now describe the ingredients that go into the proof of the theorem. The first is a general construction of a $\mathrm{Cl}(k)$-valued invariant associated with certain group actions. Specifically, suppose that we have an affine algebraic group $G$, an affine space $V$ on which $G$ acts rationally, a nonzero polynomial $P$ on $V$, and a character $\omega$ of $G$ such that $P(g x)=\omega(g)^{m} P(x)$ for some $m \geqslant 1$. Suppose also that all of these objects are defined over the ring $\mathcal{O}_{k}$. In this setting we obtain a map $S: G_{k} \backslash V_{k}^{\prime} \rightarrow \mathrm{Cl}(k)$, where $V^{\prime}$ is the complement of the hypersurface $P=0$. The construction of $S$ is described in $\S 2$.

If we take $G=\mathrm{GL}(2)$ and $V$ to be the space of binary cubic forms, with the associated discriminant polynomial and character, then it is known that the set $G_{k} \backslash V_{k}^{\prime}$ is in one-to-one correspondence with the set of isomorphism classes of separable cubic $k$-algebras. We write $A_{x}$ for the cubic $k$-algebra associated with $x \in V_{k}^{\prime}$. The correspondence $x \leftrightarrow A_{x}$ was constructed by Delone and Fadeev [DF64] by giving an explicit model for the algebra $A_{x}$ in terms of the coefficients of $x$. The key observation that makes our result possible is that the Steinitz invariant of $\mathcal{O}_{A_{x}}$ is equal to the invariant $S(x)$ described in the previous paragraph. This identification is made in $\S 3$.

In order to exploit this equality, we turn to the zeta function of the space of binary cubic forms. This remarkable meromorphic function was introduced by Shintani [Shi72] when $k=\mathbb{Q}$ as a specific example of the general theory of the zeta functions of prehomogeneous vector spaces [SS74, Yuk93]. It was studied over general numbers fields by Datskovsky and Wright in a series of papers [DW86, DW88, Wri85], and further progress has recently been made by Yukie [Yuk03]. By using this body of theory we are able to obtain the analytic properties of a family of Dirichlet series that should be

$$
\xi_{d}(s, \chi)=\sum_{[K: k]=d} \frac{\chi\left(S\left(\mathcal{O}_{K}\right)\right)}{\mathcal{N}\left(\Delta_{K / k}\right)^{s}},
$$

where $\chi$ is a character of the class group of $k$. In reality, the series we obtain are more complicated than this: each term has an additional weight factor that is itself a Dirichlet series of a simpler kind. For conceptual purposes, this complication may be ignored. With the analytic properties of $\xi_{d}(s, \chi)$ in hand, standard methods of analytic number theory allow us to extract the equidistribution theorem that is our goal. The argument we use originated in Dirichlet's proof of the infinitude of primes in an arithmetic progression. The theory of the zeta function of the space of binary cubic forms is recalled in $\S 4$ and the complications due to the extra weight factors are dealt with in $\S 5$, thus completing the proof. We would like to emphasize that Yukie's results in [Yuk03] are essential for our success. A forthcoming paper of Taniguchi will include still more precise information on the analytic properties of the zeta function. This work should appear in due course, and can serve as an alternate reference whenever we require results from [Yuk03].

In response to a question raised by the referee, we offer some remarks on error terms and related issues. Denote by $F_{3}(B)$ the set of cubic extensions $K$ of $k$ with $\mathcal{N}\left(\Delta_{K / k}\right) \leqslant B$, and by $F_{3}(\mathcal{C}, B)$ the subset of $F_{3}(B)$ consisting of fields with $S\left(\mathcal{O}_{K}\right)=\mathcal{C}$. It is known that there is a constant $\alpha>0$ such that $\left|F_{3}(B)\right|=\alpha B+o(B)$ as $B \rightarrow \infty$. In unpublished work, the second-named author improved this to $\left|F_{3}(B)\right|=\alpha B+O(B / \log (B))$. Our main result here states that $\left|F_{3}(\mathcal{C}, B)\right|=(\alpha / h) B+o(B)$, and this can be improved in the same way. However, it is expected that this is very far from the true error term. In fact, as explained in [Rob01], one expects at least that there are constants $\beta<0$ and $r<5 / 6$ such that $\left|F_{3}(B)\right|=\alpha B+\beta B^{5 / 6}+O\left(B^{r}\right)$. One can modify the heuristics leading to this proposal to take account of the Steinitz class and the result is very interesting. It is 


\section{A. C. Kable and D. J. Wright}

that $\left|F_{3}(\mathcal{C}, B)\right|=(\alpha / h) B+\beta(\mathcal{C}) B^{5 / 6}+O\left(B^{r}\right)$, where the constant $\beta(\mathcal{C})$ depends in a non-trivial way on $\mathcal{C}$. That is, while all Steinitz classes are asymptotically equally populated to first order, when the second-order terms are taken into account, some classes are systematically favored over others. The precise formula for $\beta(\mathcal{C})$ will be presented in a later paper. In recent work, Belabas et al. [BBP04] obtained the error estimate $\left|F_{3}(B)\right|=\alpha B+O\left(B^{19 / 20+\epsilon}\right)$ for any $\epsilon>0$ when $k=\mathbb{Q}$. A first step in pursuing their method in the current setting would be to extend the Delone-Fadeev correspondence in a suitable manner to all cubic algebras over a Dedekind domain.

Next let us discuss possible extensions of our results. There are prehomogeneous vector spaces that play the same role for quartic and quintic extensions of $k$ that the space of binary cubic forms plays for cubic extensions [WY92]. The invariant $S: G_{k} \backslash V_{k}^{\prime} \rightarrow \mathrm{Cl}(k)$ attached to these spaces again coincides with the Steinitz invariant of the ring of integers of the field parameterized by an orbit and so we may hope to extend our result to $d=4$ and $d=5$. For quartic extensions, the analytic theory of the associated zeta function has nearly reached the point where this will be possible, thanks again to the efforts of Yukie. For quintic extensions it will probably be some time before the same can be said. However, the standard conjectures on the analytic behavior of the zeta function of a prehomogeneous vector space also imply our theorem in that case. It seems unlikely that there is anything special about $d \leqslant 5$ and so we venture to suggest the following conjecture. By a symmetric extension of degree $d$ we mean an extension $K / k$ of degree $d$ such that the Galois group of the normal closure of $K$ over $k$ is isomorphic to $\mathfrak{S}_{d}$.

Conjecture 1.2. For any $d \geqslant 2$ the Steinitz invariant of $\mathcal{O}_{K}$ is equidistributed in $\mathrm{Cl}(k)$ as $K$ varies over the symmetric extensions of $k$ of degree $d$, ordered by $\mathcal{N}\left(\Delta_{K / k}\right)$. This remains true even if $K$ is restricted by specifying its splitting type at a finite number of places.

Beyond this there are many other quadruples $(G, V, P, \omega)$ whose orbit space $G_{k} \backslash V_{k}^{\prime}$ is in one-toone correspondence with some set of objects of arithmetic interest. For prehomogeneous examples the reader may consult [Yuk00]. There are also non-prehomogeneous examples, such as the space of binary $n$-tics for $n \geqslant 4$. It is likely that the invariant $S(x)$ will be equidistributed (with suitable weights) in $\mathrm{Cl}(k)$ as $x$ varies over $G_{k} \backslash V_{k}^{\prime}$, provided that this set is infinite. One challenge in these cases is to identify the invariant $S$ in more familiar terms and to elucidate its significance.

\section{A general construction}

In this section we define the ideal class invariant that lies at the heart of our work. To begin with we require notation for some of the standard objects of algebraic number theory. Let $k$ be a number field, $\mathcal{O}_{k}$ be its ring of integers, $\mathrm{Cl}(k)$ be its ideal class group, and $\mathfrak{M}(k), \mathfrak{M}_{\mathrm{f}}(k)$ and $\mathfrak{M}_{\infty}(k)$ be the set of places, finite places and infinite places of $k$, respectively. For $v \in \mathfrak{M}(k), k_{v}$ will denote the completion of $k$ at $v$ and $|\cdot|_{v}$ the normalized absolute value on $k_{v}$, as in [Wei74]. If $v \in \mathfrak{M}_{\mathrm{f}}(k)$ then $\mathcal{O}_{v}$ will be the ring of integers in $k_{v}, \mathcal{P}_{v}$ the maximal ideal, $q_{v}$ the cardinality of $\mathcal{O}_{v} / \mathcal{P}_{v}, \mathcal{O}_{v}^{\times}$ the units of $\mathcal{O}_{v}$ and $\varpi_{v} \in \mathcal{P}_{v}$ a fixed uniformizer. If $\mathfrak{a}$ is a fractional of $k$ whose completion at a finite place $v$ is $\mathcal{P}_{v}^{i}$ then we shall write $\operatorname{ord}_{v}(\mathfrak{a})=i$; we extend this notation to elements $\alpha \in k^{\times}$by confusing $\alpha$ with the fractional ideal it generates. The completion of $\mathfrak{a}$ at $v$ is denoted by $\mathfrak{a}_{v}$, so that $\mathfrak{a}_{v}=\mathcal{P}_{v}^{\operatorname{ord}_{v}(\mathfrak{a})}$. Each finite place $v$ of $k$ is associated with a prime ideal $\mathfrak{p}_{v}=\mathcal{O}_{k} \cap \mathcal{P}_{v}$ of $\mathcal{O}_{k}$. There is a unique homomorphism $\mathcal{N}$ from the group of fractional ideals of $k$ to $\mathbb{Q}^{\times}$such that $\mathcal{N}\left(\mathfrak{p}_{v}\right)=q_{v}$ for all $v \in \mathfrak{M}_{\mathrm{f}}(k)$; we call $\mathcal{N}$ the ideal norm. If $\mathfrak{a}$ is a non-zero ideal of $\mathcal{O}_{k}$ then $\mathcal{N}(\mathfrak{a})$ is the cardinality of the finite ring $\mathcal{O}_{k} / \mathfrak{a}$.

The adeles of $k$ will be denoted by $\mathbb{A}_{k}$, the ideles by $\mathbb{A}_{k}^{\times}$and the idele norm by $|\cdot|$. We put

$$
\mathbb{A}_{k, \infty}^{\times}=\prod_{v \in \mathfrak{M}_{\infty}(k)} k_{v}^{\times}
$$




\section{UNIFORM DISTRIBUTION}

and regard $\mathbb{A}_{k, \infty}^{\times}$as a subgroup of $\mathbb{A}_{k}^{\times}$. The class group of $k$ may be identified with the quotient of $\mathbb{A}_{k}^{\times}$by the subgroup

$$
J_{k}=k^{\times} \cdot \mathbb{A}_{k, \infty}^{\times} \cdot \prod_{v \in \mathfrak{M}_{\mathrm{f}}(k)} \mathcal{O}_{v}^{\times} .
$$

This identification is made by sending the class of the fractional ideal $\mathfrak{a}$ of $k$ to the class of the idele $\iota(\mathfrak{a})$ whose components are given by

$$
\iota(\mathfrak{a})_{v}= \begin{cases}1 & \text { if } v \in \mathfrak{M}_{\infty}(k), \\ \varpi_{v}^{\operatorname{ord}_{v}(\mathfrak{a})} & \text { if } v \in \mathfrak{M}_{\mathrm{f}}(k) .\end{cases}
$$

We shall henceforth identify $\mathrm{Cl}(k)$ with $\mathbb{A}_{k}^{\times} / J_{k}$ via $\iota$.

Let $G$ be an algebraic group, $V$ a rational representation of $G, \omega$ a rational character of $G$ and $P$ a non-zero polynomial on $V$ that satisfies the transformation law $P(g x)=\omega(g)^{m} P(x)$ for some fixed $m \geqslant 1$. Denote by $V^{\prime}$ the complement of the hypersurface $P=0$ in $V$. Assume that the quadruple $(G, V, \omega, P)$ is defined over $\mathcal{O}_{k}$. In addition, assume that if $v \in \mathfrak{M}_{\mathrm{f}}(k)$ and $x \in V_{k_{v}}^{\prime}$ then $G_{k_{v}} x \cap V_{\mathcal{O}_{v}} \neq \emptyset$. This hypothesis will be satisfied, for example, if the center of $G$ contains a split torus that acts on $V$ by a non-trivial rational character. In this situation we define

$$
\Delta_{v}(x)=\max \left\{|P(y)|_{v} \mid y \in G_{k_{v}} x \cap V_{\mathcal{O}_{v}}\right\}
$$

for $v \in \mathfrak{M}_{\mathrm{f}}(k)$ and $x \in V_{k_{v}}^{\prime}$. Note that $|P(y)|_{v}$ is bounded above for $y \in V_{\mathcal{O}_{v}}$ and that zero is the only limit point of the set $\left|k_{v}^{\times}\right|_{v}$; thus the indicated maximum is achieved, and $\Delta_{v}(x)$ is well defined. It is clear that $\Delta_{v}(x)$ depends only on the $G_{k_{v}}$-orbit of $x$. Furthermore, $\Delta_{v}(x) \leqslant 1$ for all $v \in \mathfrak{M}_{\mathrm{f}}(k)$ and $x \in V_{k_{v}}^{\prime}$.

Now let $x \in V_{k}^{\prime}$. For each finite place $v$ of $k$ we may choose $g_{x, v} \in G_{k_{v}}$ such that $\left|P\left(g_{x, v} x\right)\right|_{v}=$ $\Delta_{v}(x)$. Let $a(x)$ be the idele whose components are

$$
a(x)_{v}= \begin{cases}1 & \text { if } v \in \mathfrak{M}_{\infty}(k), \\ \omega\left(g_{x, v}\right) & \text { if } v \in \mathfrak{M}_{\mathrm{f}}(k),\end{cases}
$$

and $S(x)$ be the class of $a(x)$ in $\mathrm{Cl}(k)$. Note that $x \in V_{\Theta_{v}}$ and $|P(x)|_{v}=1$ for all but finitely many $v \in \mathfrak{M}_{\mathrm{f}}(k)$. Thus $\omega\left(g_{x, v}\right) \in \mathcal{O}_{v}^{\times}$for all but finitely many $v \in \mathfrak{M}_{\mathrm{f}}(k)$, so that $a(x)$ is well defined.

Lemma 2.1. The class $S(x) \in \mathrm{Cl}(k)$ does not depend on the choices made in its definition. If $x \in V_{k}^{\prime}$ and $g \in G_{k}$ then $S(g x)=S(x)$, so that $S$ may be regarded as a map from $G_{k} \backslash V_{k}^{\prime}$ to $\mathrm{Cl}(k)$.

Proof. The only choices made in the definition of $S(x)$ are those of $g_{x, v}$ for $v \in \mathfrak{M}_{\mathrm{f}}(k)$. Suppose that $\left(g_{x, v}\right)_{v}$ is one possible choice and $\left(g_{x, v}^{\prime}\right)_{v}$ is another. Then

$$
\left|\omega\left(g_{x, v}\right)\right|_{v}=\left(\left|P\left(g_{x, v} x\right)\right|_{v} /|P(x)|_{v}\right)^{1 / m}=\left(\Delta_{v}(x) /|P(x)|_{v}\right)^{1 / m}
$$

and similarly with $g_{x, v}^{\prime}$ in place of $g_{x, v}$. It follows that $\left|\omega\left(g_{x, v}\right)\right|_{v}=\left|\omega\left(g_{x, v}^{\prime}\right)\right|_{v}$ and so $\omega\left(g_{x, v}^{\prime}\right) \omega\left(g_{x, v}\right)^{-1} \in \mathcal{O}_{v}^{\times}$. If $a(x)$ is the idele defined with $\left(g_{x, v}\right)_{v}$ and $a^{\prime}(x)$ is the idele defined with $\left(g_{x, v}^{\prime}\right)_{v}$ then we conclude that $a^{\prime}(x) a(x)^{-1} \in J_{k}$ and so $[a(x)]=\left[a^{\prime}(x)\right]$.

Take $x \in V_{k}^{\prime}$ and $g \in G_{k}$ and suppose that $\left(g_{x, v}\right)_{v}$ is as above. As $\Delta_{v}(g x)=\Delta_{v}(x)$, the family $\left(g_{x, v} g^{-1}\right)_{v}$ has the defining property with respect to $g x$ and so $a(g x)=a(x) \omega(g)^{-1}$. However, $\omega(g) \in k^{\times}$and so $a(g x) a(x)^{-1} \in J_{k}$. Thus $[a(g x)]=[a(x)]$.

The construction of the map $S$ is rather formal and the reader might wonder what its significance is. In the next section we throw some light on this question by identifying the map $S$ associated with the space of binary cubic forms with the Steinitz invariant of the ring of integers of a cubic extension of a number field. 


\section{A. C. Kable and D. J. Wright}

\section{Binary cubic forms and the Steinitz invariant}

For the reader's convenience, we recall certain facts from the theory of modules over Dedekind domains. This theory is developed at length in [Bou65, ch. 7] and is also described in [Coh00, ch. 1] and in [FT91, ch. 2]. Let $R$ be a Dedekind domain with field of fractions $F$ and $M$ be a finitelygenerated $R$-module. Then $M$ is projective if and only if it is torsion-free. If $M$ is torsion-free then of rank $m$ then there is a fractional ideal $\mathfrak{a}$ of $F$ such that $M \cong R^{m-1} \oplus \mathfrak{a}$ and the ideal class of $\mathfrak{a}$ depends only on the isomorphism class of $M$. This ideal class is called the Steinitz invariant of $M$ and we shall denote it by $S(M)$. Note that in the Picard group of $\mathcal{O}_{k}$ we have $S(M)=\wedge^{m} M$; this interpretation of the Steinitz invariant has recently been emphasized by Khare and Prasad [KP00]. The theory we have just recalled applies in particular to the ring of integers of a number field. In this case, we have the following result [Art65].

Lemma 3.1 (Artin [Art65]). Let $k$ be a number field and $K$ a finite extension of $k$. Let $\delta_{K / k} \in k^{\times}$ be the discriminant of the trace form with respect to some $k$-basis of $K$. There is a fractional ideal $\mathfrak{a}$ of $k$ such that $\Delta_{K / k}=\left(\delta_{K / k}\right) \mathfrak{a}^{2}$ and $S\left(\mathcal{O}_{K}\right)=[\mathfrak{a}]$.

Our next task is to describe the space of binary cubic forms and its relation with the set of based cubic algebras. This subject has received detailed treatments in [DF64, WY92] and so, apart from establishing essential notation, we shall be brief. The new feature of our discussion is Proposition 3.3, which identifies the Steinitz invariant of a cubic ring in this picture.

Working initially over $\mathbb{Z}$, let $V$ be affine four-space and denote by $x_{1}, \ldots, x_{4}$ the canonical coordinates on $V$. With a point $x=\left(x_{1}, \ldots, x_{4}\right)$ in $V$ we associate the binary cubic form

$$
F_{x}\left(u_{1}, u_{2}\right)=x_{1} u_{1}^{3}+x_{2} u_{1}^{2} u_{2}+x_{3} u_{1} u_{2}^{2}+x_{4} u_{2}^{3} .
$$

The group $G=\operatorname{GL}(2)$ acts on $V$ in such a way that

$$
F_{g x}\left(u_{1}, u_{2}\right)=\operatorname{det}(g)^{-1} F_{x}\left(\left(u_{1}, u_{2}\right) g\right)
$$

and the quartic polynomial

$$
P(x)=x_{2}^{2} x_{3}^{2}+18 x_{1} x_{2} x_{3} x_{4}-4 x_{2}^{3} x_{4}-4 x_{1} x_{3}^{3}-27 x_{1}^{2} x_{4}^{2}
$$

on $V$ satisfies the transformation law

$$
P(g x)=\operatorname{det}(g)^{2} P(x)
$$

with respect to this action. With $\omega(g)=\operatorname{det}(g)$ and $m=2$, this places us in the situation of $\S 2$ and we shall employ the notation introduced there. When $k$ is a number field we obtain a map $S: G_{k} \backslash V_{k}^{\prime} \rightarrow \mathrm{Cl}(k)$ associated with the quadruple $(G, V, \omega, P)$.

Let $R$ be a commutative ring with 1 and $A$ a free $R$-module of rank three with a fixed ordered basis $e, \omega_{1}, \omega_{2}$. If $x \in V_{R}$ then we may introduce an $R$-bilinear multiplication on $A$ in such a way that it becomes a commutative, associative $R$-algebra with multiplicative identity $e$ by defining the basic products as

$$
\begin{gathered}
\omega_{1}^{2}=-x_{1} x_{3} e-x_{2} \omega_{1}+x_{1} \omega_{2}, \\
\omega_{2}^{2}=-x_{2} x_{4} e-x_{4} \omega_{1}+x_{3} \omega_{2}, \\
\omega_{1} \omega_{2}=-x_{1} x_{4} e .
\end{gathered}
$$

We denote the resulting $R$-algebra by $A_{x}$. The map $x \mapsto A_{x}$ was discovered by Delone and Fadeev [DF64] and hence we call it the Delone-Fadeev map. For brevity's sake, we define a cubic R-algebra to be a commutative, associative $R$-algebra with identity that is free of rank three as an $R$-module. The following result may be extracted from the discussion in [DF64, § 15]. 


\section{UNIFORM DISTRIBUTION}

Lemma 3.2 (Delone-Fadeev [DF64]). The isomorphism class of $A_{x}$ depends only on the $G_{R^{-o r b i t}}$ of $x$ in $V_{R}$. The discriminant of the trace form of $A_{x}$ with respect to the basis $e, \omega_{1}, \omega_{2}$ is $P(x)$. The set $G_{R} \backslash V_{R}^{\prime}$ is in one-to-one correspondence with the set of isomorphism classes of separable cubic $R$-algebras via the Delone-Fadeev map.

Let $k$ be a number field. If $K$ is a separable cubic $k$-algebra then $K$ is isomorphic to a direct sum of number fields and so we may make sense of the notation $\mathcal{O}_{K}$ as the direct sum of the rings of integers in each of these fields. Then $\mathcal{O}_{K}$ is a torsion-free $\mathcal{O}_{k}$-module and so the Steinitz invariant $S\left(\mathcal{O}_{K}\right)$ is defined. Note that if $K \cong k^{3}$ then $S\left(\mathcal{O}_{K}\right)$ is the identity in $\mathrm{Cl}(k)$ and if $K \cong k \oplus E$, with $E$ a quadratic extension of $k$, then $S\left(\mathcal{O}_{K}\right)=S\left(\mathcal{O}_{E}\right)$. Observe that Lemma 3.1 extends without change to this setting.

Take $v \in \mathfrak{M}(k)$. As the set of extensions of $k_{v}$ of fixed degree is finite, $G_{k_{v}} \backslash V_{k_{v}}^{\prime}$ is finite. It will be convenient to choose specific representatives for each of the orbits in this set. Let $n(v)$ be the cardinality of $G_{k_{v}} \backslash V_{k_{v}}^{\prime}$. If $v \in \mathfrak{M}_{\infty}(k)$ then choose representatives $w_{i, v}, 1 \leqslant i \leqslant n(v)$, for the orbits in $G_{k_{v}} \backslash V_{k_{v}}^{\prime}$ such that $\left|P\left(w_{i, v}\right)\right|_{v}=1$. Note that $n(v)=1$ if $v$ is a complex place and $n(v)=2$ if $v$ is a real place. Suppose now that $v \in \mathfrak{M}_{\mathrm{f}}(k)$, take $x \in G_{k_{v}} \backslash V_{k_{v}}^{\prime}$ and let $A_{x}$ be the associated separable cubic $k_{v}$-algebra. Then $A_{x}$ is isomorphic to a direct sum of local fields and there is unique maximal compact subring $R_{x}$ in $A_{x}$. The ring $R_{x}$ is in fact a cubic $\mathcal{O}_{v}$-algebra and there is an orbit $G_{\mathcal{O}_{v}} w$ in $G_{\mathcal{O}_{v}} \backslash V_{\mathcal{O}_{v}}^{\prime}$ that corresponds to $R_{x}$ under the Delone-Fadeev map for $\mathcal{O}_{v}$. As every $\mathcal{O}_{v}$-subalgebra of $A_{x}$ is contained in $R_{x}, G_{\mathcal{O}_{v}} w$ is the unique $G_{\mathcal{O}_{v}}$-orbit on which $|P(y)|_{v}$ achieves its maximum value for $y \in G_{k_{v}} x \cap V_{\mathcal{O}_{v}}$. In the notation of $\S 2$ this means that $\Delta_{v}(x)=|P(w)|_{v}$ and, moreover, that this equality determines the $G_{\mathcal{O}_{v}}$-orbit of $w$. We now choose representatives $w_{i, v}, 1 \leqslant i \leqslant n(v)$, for the orbits in $G_{k_{v}} \backslash V_{k_{v}}^{\prime}$ that satisfy $\Delta_{v}\left(w_{i, v}\right)=\left|P\left(w_{i, v}\right)\right|_{v}$. With orbital representatives fixed at all $v \in \mathfrak{M}(k)$, we introduce one more piece of notation: if $x \in V_{k_{v}}^{\prime}$ then let $i_{v}(x)$ be the index such that $x \in G_{k_{v}} w_{i_{v}(x), v}$.

Proposition 3.3. Let $k$ be a number field, $x \in V_{k}^{\prime}$ and $K$ the separable cubic $k$-algebra corresponding to $x$. Then $S\left(\mathcal{O}_{K}\right)=S(x)$.

Proof. For each $v \in \mathfrak{M}_{\mathrm{f}}(k)$ choose $g_{x, v} \in G_{k_{v}}$ such that $g_{x, v} x=w_{i_{v}(x), v}$. Let $a(x)$ be the idele whose infinite components are all 1 and whose component at a finite place $v$ is $\operatorname{det}\left(g_{x, v}\right)$. The choice of orbital representatives $w_{i, v}$ made above is consistent with the definition of $S$ and so $S(x)=[a(x)]$. The cubic $\mathcal{O}_{v}$-algebra $R_{v}=\mathcal{O}_{v} \otimes_{\mathcal{O}_{k}} \mathcal{O}_{K}$ is the maximal compact subring of the cubic $k_{v}$-algebra $k_{v} \otimes_{k} K$. As such, $R_{v}$ corresponds to $w_{i_{v}(x), v}$ under the Delone-Fadeev map, and it follows from Lemma 3.2 that the discriminant ideal of $R_{v}$ over $\mathcal{O}_{v}$ is generated by $P\left(w_{i_{v}(x), v}\right)$. This ideal coincides with the completion of $\Delta_{K / k}$ at $v$. We have $P\left(w_{i_{v}(x), v}\right)=\operatorname{det}\left(g_{x, v}\right)^{2} P(x)$ and so if $\mathfrak{a}(x)$ is the fractional ideal of $k$ corresponding to the idele $a(x)$ then $\Delta_{K / k}=(P(x)) \mathfrak{a}(x)^{2}$. By Lemma 3.2, $P(x)$ is the discriminant of the trace form with respect to some $k$-basis of $K$. It follows from Lemma 3.1, or its extension to separable algebras, that $S\left(\mathcal{O}_{K}\right)=[\mathfrak{a}(x)]$.

\section{The zeta function}

In this section we describe the zeta function of the space of binary cubic forms and its connection to the problem considered here. As usual, we must begin by introducing some further notation and recalling various standard facts.

Let $k$ be a number field. A continuous homomorphism $\omega: \mathbb{A}_{k}^{\times} / k^{\times} \rightarrow \mathbb{C}^{\times}$will be referred to as an idele class character of $k$. The group of all idele class characters of $k$ will be denoted by $\Omega(k)$. Included in this group are the principal idele class characters $\omega_{s}$ for $s \in \mathbb{C}$, defined by $\omega_{s}(a)=|a|^{s}$. Let $\mathbb{A}_{k}^{1}=\left\{a \in \mathbb{A}_{k}^{\times}|| a \mid=1\right\}$ be the kernel of the idele norm. Associated with the idele norm there 


\section{A. C. Kable and D. J. Wright}

is a short exact sequence

$$
\{1\} \longrightarrow \mathbb{A}_{k}^{1} \longrightarrow \mathbb{A}_{k}^{\times} \longrightarrow \mathbb{R}_{+} \longrightarrow\{1\},
$$

where $\mathbb{R}_{+}$denotes the multiplicative group of positive real numbers. This sequence is split and we may choose a specific splitting $\lambda \mapsto \underline{\lambda}$ such that $\underline{\lambda}_{v}=1$ for all $v \in \mathfrak{M}_{\mathrm{f}}(k)$. Once this splitting is chosen we obtain an isomorphism

$$
\mathbb{A}_{k}^{\times} \cong \mathbb{R}_{+} \times \mathbb{A}_{k}^{1} .
$$

If $a \in \mathbb{A}_{k}$ then let $(|a|, \tilde{a}) \in \mathbb{R}_{+} \times \mathbb{A}_{k}^{1}$ be the pair corresponding to $a$ under the isomorphism (1). We also have an isomorphism

$$
\mathbb{A}_{k}^{\times} / k^{\times} \cong \mathbb{R}_{+} \times \mathbb{A}_{k}^{1} / k^{\times}
$$

induced by (1).

Let $\Omega^{1}(k)$ be the group of continuous homomorphisms from $\mathbb{A}_{k}^{1} / k^{\times}$to $\mathbb{C}^{\times}$; note that, since $\mathbb{A}_{k}^{1} / k^{\times}$is compact, the image of any such homomorphism actually lies in $S^{1}=\{z \in \mathbb{C}|| z \mid=1\}$. Corresponding to the isomorphism (2) we obtain an isomorphism $\Omega(k) \cong \mathbb{C} \times \Omega^{1}(k)$. The character $\omega \in \Omega(k)$ corresponds under this isomorphism to the pair $\left(s(\omega),\left.\omega\right|_{\mathbb{A}_{k}^{1} / k^{\times}}\right)$, where $s(\omega)$ is the unique complex number such that $\omega(\underline{\lambda})=\lambda^{s(\omega)}$. If $\omega \in \Omega^{1}(k)$ then let $\tilde{\omega} \in \Omega(k)$ be the character defined by $\tilde{\omega}(a)=\omega(\tilde{a})$. Note that $s(\tilde{\omega})=0$ for all $\omega \in \Omega^{1}(k)$. As $J_{k}$ contains the image of $\lambda \mapsto \underline{\lambda}$, the inclusion $\mathbb{A}_{k}^{1} \rightarrow \mathbb{A}_{k}$ induces an isomorphism

$$
\mathrm{Cl}(k) \cong \mathbb{A}_{k}^{1} / J_{k}^{1},
$$

where $J_{k}^{1}=J_{k} \cap \mathbb{A}_{k}^{1}$. Thus, we may identify the characters of the finite group $\mathrm{Cl}(k)$ with the characters of $\mathbb{A}_{k}^{1}$ that are trivial on $J_{k}^{1}$.

Denote by $G_{\mathbb{A}}$, respectively $V_{\mathbb{A}}$, be the adele points of $G$, respectively $V$, as a group over $k$. Let $U_{v} \subset G_{k_{v}}$ be the orthogonal group if $v$ is a real place of $k$, the unitary group if $v$ is a complex place of $k$, and $G_{\mathcal{O}_{v}}$ if $v$ is a finite place of $k$. Then

$$
U=\prod_{v \in \mathfrak{M}(k)} U_{v} \subset G_{\mathbb{A}}
$$

is a maximal compact subgroup of $G_{\mathbb{A}}$. The image of the determinant map restricted to $U$ is $\mathbb{A}_{k}^{0}=\left\{\left.a \in \mathbb{A}_{k}|| a_{v}\right|_{v}=1 \forall v \in \mathfrak{M}(k)\right\}$. Let $\mathcal{S}\left(V_{\mathbb{A}}\right)$ be the space of Schwartz-Bruhat functions on $V_{\mathbb{A}}$ and $\mathcal{S}_{0}\left(V_{\mathbb{A}}\right) \subset \mathcal{S}\left(V_{\mathbb{A}}\right)$ be the subspace consisting of $U$-invariant functions.

In [DW86, Wri85], specific Haar measures were fixed on $\mathbb{A}_{k}, \mathbb{A}_{k}^{\times}$and $G_{\mathbb{A}}$, as well as on the local factors of each of these groups. We employ the same measures here, albeit with a simplified notation that should be clear in context. From these basic choices, we also obtain Haar measures on $V_{\mathbb{A}}$ and its local factors and on quotient spaces such as $G_{\mathbb{A}} / G_{k}$.

Let $X \subset V_{k}^{\prime}$ be a $G_{k}$-invariant set, $\omega \in \Omega(k)$ and $\Phi \in \mathcal{S}_{0}\left(V_{\mathbb{A}}\right)$. We define the associated zeta function to be

$$
Z(\omega, \Phi ; X)=\int_{G_{\mathbb{A}} / G_{k}} \omega(\operatorname{det}(g)) \sum_{x \in X} \Phi(g x) d g .
$$

Note that $Z(\omega, \Phi ; X) \equiv 0$ unless $\omega$ is trivial on $\mathbb{A}_{k}^{0} \cdot k^{\times} / k^{\times}$. If $x \in V_{k}^{\prime}$ then let $A_{x}$ be the cubic $k$-algebra corresponding to $x$ under the Delone-Fadeev map. We have to consider the $G_{k}$-invariant sets

$$
\begin{gathered}
X_{2}=\left\{x \in V_{k}^{\prime} \mid A_{x} \cong k \oplus E \text { with } E \text { a field }\right\}, \\
X_{3}=\left\{x \in V_{k}^{\prime} \mid A_{x} \text { is a field }\right\} .
\end{gathered}
$$




\section{UNIFORM DISTRIBUTION}

Define functionals $\Sigma_{2}$ and $\Sigma_{3}$ on $\mathcal{S}_{0}\left(V_{\mathbb{A}}\right)$ by

$$
\Sigma_{2}(\Phi)=\int_{\mathbb{A}_{k}^{\times}} \int_{\mathbb{A}_{k}} \int_{\mathbb{A}_{k}}|t|^{2} \Phi\left(0, t, y_{1}, y_{2}\right) d y_{1} d y_{2} d^{\times} t
$$

and

$$
\Sigma_{3}(\Phi)=\int_{V_{\mathbb{A}}} \Phi(x) d x
$$

The following result summarizes some of the analytic properties of the zeta functions $Z\left(\omega, \Phi ; X_{2}\right)$ and $Z\left(\omega, \Phi ; X_{3}\right)$ in a form convenient for our applications. When discussing the convergence of integrals we use the phrase normally convergent to mean absolutely and locally uniformly convergent.

Proposition 4.1. Let $\chi \in \Omega^{1}(k), \Phi \in \mathcal{S}_{0}\left(V_{\mathbb{A}}\right)$ and $s \in \mathbb{C}$ and put

$$
Z_{d}(s, \chi, \Phi)=Z\left(\omega_{s} \tilde{\chi}, \Phi ; X_{d}\right)
$$

for $d=2,3$. The integral defining $Z_{d}(s, \chi, \Phi)$ is normally convergent in the region $\operatorname{re}(s)>2$. The resulting holomorphic function has a meromorphic continuation to the region $\operatorname{re}(s)>5 / 3$. If $\chi$ is non-trivial then this continuation is, in fact, holomorphic. If $\chi=\chi_{0}$ is trivial then the only possible singularity of $Z_{d}\left(s, \chi_{0}, \Phi\right)$ in the region re $(s)>5 / 3$ is a simple pole at $s=2$ with residue $C_{d} \Sigma_{d}(\Phi)$, where $C_{2}$ and $C_{3}$ are positive constants depending only on $k$.

Proof. The analytic properties of $Z\left(\omega, \Phi ; V_{k}^{\prime}\right)$ were studied in detail in [Wri85]. If $x_{0} \in V_{k}^{\prime}$ is a point corresponding to the cubic $k$-algebra $k^{3}$ then we have $V_{k}^{\prime}=X_{3} \cup X_{2} \cup G_{k} x_{0}$ and $Z\left(\omega, \Phi ; G_{k} x_{0}\right)$ is easily understood. Thus, if one can obtain the analytic properties of either $Z\left(\omega, \Phi ; X_{2}\right)$ or $Z\left(\omega, \Phi ; X_{3}\right)$ then those of the other will follow. A direct analysis of $Z\left(\omega_{s}, \Phi ; X_{2}\right)$ is achieved in [Yuk03]. Although this work does not consider $Z\left(\omega_{s} \tilde{\chi}, \Phi ; X_{2}\right)$ for non-trivial $\chi$, the proof turns on the estimation of the region of absolute convergence of certain integrals connected with $Z\left(\omega_{s}, \Phi ; X_{2}\right)$ and, as $|\tilde{\chi}(a)|=1$ for all $a \in \mathbb{A}_{k}^{\times}$, it is a straightforward matter to modify the arguments to cover the more general case.

Every $\Phi \in \mathcal{S}_{0}\left(V_{\mathbb{A}}\right)$ is a finite sum of functions that factor as a product over $v \in \mathfrak{M}(k)$ of local Schwartz-Bruhat functions. We assume henceforth that all $\Phi$ that we consider have a factorization $\Phi=\bigotimes_{v} \Phi_{v}$. Note that $\Phi_{v}$ is invariant under the group $U_{v}$. If $v \in \mathfrak{M}_{\mathrm{f}}(k)$ then denote by $\Phi_{0, v}$ the characteristic function of the compact open set $V_{\mathcal{O}_{v}}$. If $\Phi \in \mathcal{S}_{0}\left(V_{\mathbb{A}}\right)$ then $\Phi_{v}=\Phi_{0, v}$ for all but finitely many places $v$. If $\omega \in \Omega(k)$ then the pullback of $\omega$ to $\mathbb{A}_{k}^{\times}$has a factorization $\omega=\bigotimes_{v} \omega_{v}$. The local character $\omega_{v}$ is unramified for all but finitely many $v$. We have $\left|\omega_{v}(x)\right|=|x|_{v}^{\mathrm{re}(s(\omega))}$ for all $v \in \mathfrak{M}(k)$ and all $x \in k_{v}^{\times}$. When we are discussing a single place of $k$ we may denote the exponent in this identity by re $\left(\omega_{v}\right)$, which is defined without regard to a global character of which $\omega_{v}$ is a factor.

Recall that in $\S 3$ we chose standard local orbital representatives $w_{i, v} \in V_{k_{v}}^{\prime}$ subject to certain conditions. If $x \in V_{k}^{\prime}$ then, for all $v \in \mathfrak{M}(k), x \in G_{k_{v}} w_{i_{v}(x), v}$. For $x \in V_{k}^{\prime}, \omega \in \Omega(k)$ and $\Phi \in \mathcal{S}_{0}\left(V_{\mathbb{A}}\right)$ we define

$$
\Xi_{x}(\omega, \Phi)=\prod_{v \in \mathfrak{M}(k)} \Xi_{x, v}\left(\omega_{v}, \Phi_{v}\right)
$$

where

$$
\Xi_{x, v}\left(\omega_{v}, \Phi_{v}\right)=\int_{G_{k_{v}}} \omega_{v}\left(\operatorname{det}\left(g_{v}\right)\right) \Phi_{v}\left(g_{v} w_{i_{v}(x), v}\right) d g_{v} .
$$

Note that $\Xi_{x}(\omega, \Phi)$ depends on $x$ only through its $G_{k}$-orbit. In order to justify these definitions, we give a preliminary convergence result.

Proposition 4.2. The integral defining $\Xi_{x, v}\left(\omega_{v}, \Phi_{v}\right)$ is normally convergent in the region $\operatorname{re}\left(\omega_{v}\right)>$ $1 / 3$. After possibly discarding a finite number of vanishing factors, the product that defines $\Xi_{x}(\omega, \Phi)$ is absolutely convergent in the region $\operatorname{re}(s(\omega))>1$. 


\section{A. C. Kable and D. J. Wright}

Proof. The statements are a consequence of [DW86, Corollaries 3.1 and 3.2 and Theorem 3.1], together with the observations recorded above.

The following result establishes the essential connection between $Z_{d}(s, \chi, \Phi)$ and the distribution of the Steinitz invariant of degree $d$ extensions of $k$ in the class group. For each $x \in V_{k}^{\prime}$ let $o(x)$ be the cardinality of the isotropy group of $x$ in $G_{k}$. The number $o(x)$ is also the cardinality of the group of automorphisms of the cubic $k$-algebra $A_{x}$ (see, for example, [DW86]); in particular, $1 \leqslant o(x) \leqslant 6$.

Proposition 4.3. Let $\chi$ be a character of $\mathrm{Cl}(k)$ and regard $\chi$ equivalently as an element of $\Omega^{1}(k)$. Let $\Phi \in \mathcal{S}_{0}\left(V_{\mathbb{A}}\right)$ and $s \in \mathbb{C}$ with $\operatorname{re}(s)>2$. There is a positive constant $C_{0}$, depending only on $k$, such that

$$
Z_{d}(s, \chi, \Phi)=C_{0} \sum_{x \in G_{k} \backslash X_{d}} o(x)^{-1} \chi\left(S\left(\mathcal{O}_{A_{x}}\right)\right) \Xi_{x}\left(\omega_{s} \tilde{\chi}, \Phi\right) \mathcal{N}\left(\Delta_{A_{x} / k}\right)^{-s / 2}
$$

for $d=2,3$.

Proof. By unfolding the integral against the sum in the definition of $Z_{d}(s, \chi, \Phi)$ we obtain

$$
Z_{d}(s, \chi, \Phi)=\sum_{x \in G_{k} \backslash X_{d}} o(x)^{-1} \int_{G_{\mathbb{A}}}|\operatorname{det}(g)|^{s} \tilde{\chi}(\operatorname{det}(g)) \Phi(g x) d g .
$$

Each term in this series may be written as a product of local factors. The Haar measure on $G_{\mathbb{A}}$ is necessarily proportional to the product of the Haar measures on $G_{k_{v}}$ and so

$$
Z_{d}(s, \chi, \Phi)=C_{0} \sum_{x \in G_{k} \backslash X_{d}} o(x)^{-1} \prod_{v \in \mathfrak{M}(k)} \int_{G_{k_{v}}}\left|\operatorname{det}\left(g_{v}\right)\right|_{v}^{s} \tilde{\chi}_{v}\left(\operatorname{det}\left(g_{v}\right)\right) \Phi_{v}\left(g_{v} x\right) d g_{v},
$$

where $C_{0}$ depends only on $k$. For each $x \in X_{d}$ and $v \in \mathfrak{M}(k)$ we may choose $g_{x, v} \in G_{k_{v}}$ such that $g_{x, v} x=w_{i_{v}(x), v}$. Let $a(x)$ be the idele whose component at $v$ is $\operatorname{det}\left(g_{x, v}\right)$. After making the change of variable $g_{v} \mapsto g_{v} g_{x, v}$ in each of the local integrals, we arrive at

$$
Z_{d}(s, \chi, \Phi)=C_{0} \sum_{x \in G_{k} \backslash X_{d}} o(x)^{-1}|a(x)|^{s} \tilde{\chi}(a(x)) \Xi_{x}\left(\omega_{s} \tilde{\chi}, \Phi\right) .
$$

As $\mathbb{A}_{k, \infty}^{\times} \subset J_{k}$, it follows from the definition that $a(x)$ lies in the class $S(x)$ in $\mathbb{A}_{k}^{\times} / J_{k}$. By Proposition 3.3 this is also the class $S\left(\mathcal{O}_{A_{x}}\right)$ and so $\tilde{\chi}(a(x))=\chi\left(S\left(\mathcal{O}_{A_{x}}\right)\right)$. Furthermore, we have $P\left(w_{i_{v}(x), v}\right)=\operatorname{det}\left(g_{x, v}\right)^{2} P(x)$ and so

$$
|a(x)|=\prod_{v \in \mathfrak{M}(k)}\left|P\left(w_{i_{v}(x), v}\right)\right|_{v}^{1 / 2}|P(x)|_{v}^{-1 / 2}=\prod_{v \in \mathfrak{M}(k)}\left|P\left(w_{i_{v}(x), v}\right)\right|_{v}^{1 / 2},
$$

where we have applied the Artin product formula to $P(x) \in k^{\times}$to obtain the second equality. The representatives $w_{i_{v}(x), v}$ were chosen so that $\left|P\left(w_{i_{v}(x), v}\right)\right|_{v}=1$ if $v \in \mathfrak{M}_{\infty}(k)$ and $w_{i_{v}(x), v}$ corresponds under the Delone-Fadeev map to the maximal order $R_{x, v}=\mathcal{O}_{v} \otimes_{\mathcal{O}_{k}} \mathcal{O}_{A_{x}}$ if $v \in \mathfrak{M}_{\mathrm{f}}(k)$. If $v \in \mathfrak{M}_{\mathrm{f}}(k)$ then it follows from Lemma 3.2 that $P\left(w_{i_{v}(x), v}\right) \mathcal{O}_{v}$ is the discriminant ideal of $R_{x, v}$. Thus

$$
|a(x)|=\prod_{v \in \mathfrak{M}_{\mathrm{f}}(k)}\left|P\left(w_{i_{v}(x), v}\right)\right|_{v}^{1 / 2}=\mathcal{N}\left(\Delta_{A_{x} / k}\right)^{-1 / 2}
$$

and we obtain the required identity.

\section{The filtration process}

If $\Xi_{x}\left(\omega_{s} \tilde{\chi}, \Phi\right)$ were absent from the expression for $Z_{d}(s, \chi, \Phi)$ given in Proposition 4.3 then our main result would follow from Proposition 4.1, a Tauberian theorem for Dirichlet series and the 


\section{UNIFORM DISTRIBUTION}

orthogonality relations for the characters of $\mathrm{Cl}(k)$. The purpose of this section is to make the technical modifications to this simple argument necessitated by the presence of $\Xi_{x}\left(\omega_{s} \tilde{\chi}, \Phi\right)$. We refer to these modifications as the filtration process. This process, first described in [DW88], has previously been used to obtain density results from series such as $Z_{d}\left(s, \chi_{0}, \Phi\right)$. As we must deal with $Z_{d}(s, \chi, \Phi)$, with $\chi$ not necessarily trivial, we require a minor variant of the usual argument.

We have to consider the set of extensions of $k$ whose completion at a finite number of places is fixed. To this end, let $T \subset \mathfrak{M}(k)$ be a finite set. Recall that $n(v)$ denotes the cardinality of the set $G_{k_{v}} \backslash V_{k_{v}}^{\prime}$ and that we have indexed the $G_{k_{v}}$-orbits by choosing representatives $w_{i, v}, 1 \leqslant i \leqslant n(v)$, for them. A $T$-type $\alpha$ will be a map $\alpha: T \rightarrow \mathbb{N}$ such that $1 \leqslant \alpha(v) \leqslant n(v)$ for each $v \in T$. If $\alpha$ is a $T$-type and $x \in V_{k}^{\prime}$ then we write $x \approx \alpha$ if $i_{v}(x)=\alpha(v)$ for all $v \in T$. Thus, $x \approx \alpha$ if the completion of the cubic $k$-algebra $A_{x}$ at each $v \in T$ is the cubic $k_{v}$-algebra corresponding to the orbit indexed by $\alpha(v)$. If $T \subset T^{\prime}, \alpha$ is a $T$-type and $\alpha^{\prime}$ is a $T^{\prime}$-type then we write $\alpha^{\prime} \mid T=\alpha$ if $\alpha^{\prime}(v)=\alpha(v)$ for all $v \in T$.

If $T \subset \mathfrak{M}(k)$ is a finite set, $\alpha$ is a $T$-type, $\mathcal{C} \in \mathrm{Cl}(k)$ and $B>0$ then the sets

$$
\begin{gathered}
X_{d}(\alpha)=\left\{x \in X_{d} \mid x \approx \alpha\right\}, \\
X_{d}(\alpha, B)=\left\{x \in X_{d} \mid x \approx \alpha, \mathcal{N}\left(\Delta_{A_{x} / k}\right) \leqslant B\right\}, \\
X_{d}(\alpha, \mathcal{C})=\left\{x \in X_{d} \mid x \approx \alpha, S\left(\mathcal{O}_{A_{x}}\right)=\mathcal{C}\right\}, \\
X_{d}(\alpha, \mathcal{C}, B)=X_{d}(\alpha, B) \cap X_{d}(\alpha, \mathcal{C})
\end{gathered}
$$

are $G_{k}$-invariant. The quotient sets $G_{k} \backslash X_{d}(\alpha, B)$ and $G_{k} \backslash X_{d}(\alpha, \mathcal{C}, B)$ are finite because of the wellknown theorem on the finiteness of the set of number fields with bounded discriminant.

For $v \in \mathfrak{M}(k), 1 \leqslant i \leqslant n(v), \omega_{v}$ a character of $k_{v}^{\times}$and $\Phi_{v} \in \mathcal{S}\left(V_{k_{v}}\right)$ we let

$$
Z_{i, v}\left(\omega_{v}, \Phi_{v}\right)=\int_{G_{k_{v}}} \omega_{v}\left(\operatorname{det}\left(g_{v}\right)\right) \Phi_{v}\left(g_{v} w_{i, v}\right) d g_{v}
$$

Of course, $\Xi_{x, v}\left(\omega_{v}, \Phi_{v}\right)=Z_{i_{v}(x), v}\left(\omega_{v}, \Phi_{v}\right)$, but it will be convenient to discuss these functions without reference to any point $x \in V_{k}^{\prime}$.

LEMma 5.1. Let $v \in \mathfrak{M}(k), 1 \leqslant i \leqslant n(v)$, and $\omega_{v}^{*}$ be an unramified character of $k_{v}^{\times}$. There exists a $U_{v}$-invariant function $\Phi_{v} \in \mathcal{S}\left(V_{k_{v}}\right)$ such that the support of $\Phi_{v}$ is contained in $G_{k_{v}} w_{i, v}, Z_{i, v}\left(\omega_{v}, \Phi_{v}\right)$ is an entire function of $\omega_{v}$ and $Z_{i, v}\left(\omega_{v}^{*}, \Phi_{v}\right) \neq 0$.

Proof. Start with a positive Schwartz function of sufficiently small compact support in the orbit and then average it over $U_{v}$.

For $v \in \mathfrak{M}(k)$ and $\Phi_{v} \in \mathcal{S}\left(V_{k_{v}}\right)$ a $U_{v}$-invariant function let

$$
\Sigma_{2, v}\left(\Phi_{v}\right)=\int_{k_{v}^{\times}} \int_{k_{v}} \int_{k_{v}}\left|t_{v}\right|_{v}^{2} \Phi_{v}\left(0, t_{v}, y_{1, v}, y_{2, v}\right) d y_{1, v} d y_{2, v} d^{\times} t_{v}
$$

and

$$
\Sigma_{3, v}\left(\Phi_{v}\right)=\int_{V_{k_{v}}} \Phi_{v}\left(x_{v}\right) d x_{v}
$$

There are positive constants $C_{2}^{\prime}$ and $C_{3}^{\prime}$ such that if $\Phi \in \mathcal{S}_{0}\left(V_{\mathbb{A}}\right)$ and $\Phi=\otimes_{v} \Phi_{v}$ then

$$
\Sigma_{d}(\Phi)=C_{d}^{\prime} \prod_{v \in \mathfrak{M}(k)} \Sigma_{d, v}\left(\Phi_{v}\right)
$$

for $d=2,3$. The constant $C_{d}^{\prime}$ is the constant of proportionality between the global measures and the product of the local measures.

In order to state the next lemma, it will be convenient to introduce an assumption on the standard representatives $w_{i, v}$ which has not been needed so far. We assume that the list $w_{1, v}, \ldots, w_{n(v), v}$ 


\section{A. C. Kable and D. J. Wright}

is so arranged that the elements corresponding to cubic $k_{v}$-algebras that are fields come at the end. Thus, there is a number $n_{2}(v) \leqslant n(v)$ such that $w_{i, v}$ corresponds to a field if and only if $i>n_{2}(v)$. The point of this is that if $x \in X_{2}$ then $1 \leqslant i_{v}(x) \leqslant n_{2}(v)$. If $T \subset \mathfrak{M}(k)$ is a finite set and $\alpha$ is a $T$-type then we say that $\alpha$ is a quadratic T-type if $\alpha(v) \leqslant n_{2}(v)$ for all $v \in T$.

Lemma 5.2. Let $v \in \mathfrak{M}(k)$. There are positive constants $b_{i, v}$, for $1 \leqslant i \leqslant n(v)$, and $c_{i, v}$, for $1 \leqslant i \leqslant n_{2}(v)$, such that

$$
Z_{i, v}\left(|\cdot|_{v}^{2}, \Phi_{v}\right)=b_{i, v} \Sigma_{3, v}\left(\Phi_{v}\right)
$$

and

$$
Z_{i, v}\left(|\cdot|_{v}^{2}, \Phi_{v}\right)=c_{i, v} \Sigma_{2, v}\left(\Phi_{v}\right)
$$

whenever $\Phi_{v} \in \mathcal{S}\left(V_{k_{v}}\right)$ is a $U_{v}$-invariant function whose support is contained in $G_{k_{v}} w_{i, v}$.

Proof. The first identity is [DW86, (2.4)]. The second follows from Theorem 5.2 of the same reference.

For $v \in \mathfrak{M}(k)$ we define

$$
E_{2, v}=\sum_{i=1}^{n_{2}(v)} c_{i, v}^{-1}
$$

and

$$
E_{3, v}=\sum_{i=1}^{n(v)} b_{i, v}^{-1} .
$$

Lemma 5.3. Let $v \in \mathfrak{M}_{\mathrm{f}}(k), 1 \leqslant i \leqslant n(v)$ and $\omega_{v}$ be an unramified character of $k_{v}^{\times}$that takes its values in $S^{1}$. As before, let $\Phi_{0, v}$ denote the characteristic function of $V_{\Theta_{v}}$. There are constants $\left\{a_{i, v, n}\right\}_{n=0}^{\infty}$ such that

$$
Z_{i, v}\left(\left.\omega_{v}|\cdot|\right|_{v} ^{s}, \Phi_{0, v}\right)=\sum_{n=0}^{\infty} a_{i, v, n} \omega_{v}\left(\varpi_{v}\right)^{n} q_{v}^{-n s}
$$

for $\operatorname{re}(s)>1 / 3$. We have $a_{i, v, 0}=1$ and $a_{i, v, n} \geqslant 0$ for all $n$.

Proof. The local integral defining $Z_{i, v}\left(\left.\omega_{v}|\cdot|\right|_{v} ^{s}, \Phi_{0, v}\right)$ was evaluated for all $i$ and $v \in \mathfrak{M}_{\mathrm{f}}(k)$ in [DW86, Theorem 3.1]. The results are conveniently summarized in [DW88, § 2]. The claims follow by inspection. In [Yuk03, $\S 6$ ] the same result is proved by a simpler method when $v$ is neither dyadic nor triadic. As we shall remark in the proof of Theorem 5.5, this would in fact suffice for our application.

Lemma 5.4. For each $v \in \mathfrak{M}_{\mathrm{f}}(k)$ there is a series

$$
L_{v}(s)=\sum_{n=0}^{\infty} l_{v, n} q_{v}^{-n s},
$$

convergent for $\mathrm{re}(s)>1 / 3$, such that $l_{v, 0}=1$ and $a_{i, v, n} \leqslant l_{v, n}$ for all $1 \leqslant i \leqslant n(v)$. These series may be chosen so that the product $\prod_{v \in \mathfrak{M}_{\mathrm{f}}(k)} L_{v}(s)$ is absolutely convergent in the region $\mathrm{re}(s)>1$.

Proof. It follows from the discussion in $[\mathrm{DW} 88, \S 2]$ that we may take $L_{v}(s)$ to be $Z_{i, v}\left(|\cdot|_{v}^{s}, \Phi_{0, v}\right)$ for that index $i$ corresponding to the cubic $k_{v}$-algebra $k_{v}^{3}$. A similar result is proved in [Yuk03, $\S 6]$ under the restriction that $v$ be neither dyadic nor triadic, and again this would suffice for our application. 


\section{UNIFORM DISTRIBUTION}

Theorem 5.5. Let $h$ be the class number of $k$ and $\mathcal{C} \in \mathrm{Cl}(k)$. Choose a finite set $T_{0} \subset \mathfrak{M}(k)$ and a $T_{0}$-type $\alpha$. Let $d=2$ or 3 and assume that $\alpha$ is a quadratic $T_{0}$-type if $d=2$. We have

$$
\lim _{B \rightarrow \infty} B^{-1} \sum_{x \in G_{k} \backslash X_{d}(\alpha, \mathcal{C}, B)} o(x)^{-1}=\frac{1}{h} \lim _{B \rightarrow \infty} B^{-1} \sum_{x \in G_{k} \backslash X_{d}(\alpha, B)} o(x)^{-1} .
$$

Proof. If $T_{1} \subset \mathfrak{M}(k)$ is a finite set containing $T_{0}$ then the $T_{0}$-type $\alpha$ extends in various ways to a $T_{1}$-type. Suppose that we can obtain the claim with $T_{1}$ in place of $T_{0}$. Then by summing over all of the extensions of $\alpha$ to a $T_{1}$-type we may obtain the original claim. Thus, we may suppose without loss of generality that $T_{0}$ contains the infinite places. Note that we could also assume that $T_{0}$ contains all the dyadic and triadic places, so that the full strength of Lemmas 5.3 and 5.4 is not really needed.

Henceforth, $T_{0}$ will be a finite set of places containing $\mathfrak{M}_{\infty}(k), \alpha$ will be a $T_{0}$-type, $T$ will be a finite set of places containing $T_{0}$ and $\beta$ will be a $T$-type extending $\alpha$. If $d=2$ then we assume, in addition, that $\beta$ is a quadratic $T$-type. For $s \in \mathbb{C}, \omega \in \Omega(k)$ and $x \in V_{k}^{\prime}$ we define

$$
L_{T}(s)=\prod_{v \notin T} L_{T}(s)
$$

and

$$
\Xi_{x, T}(\omega)=\prod_{v \notin T} \Xi_{x, v}\left(\omega_{v}, \Phi_{0, v}\right)
$$

Let $\chi$ be a character of $\mathrm{Cl}(k)$ and regard $\chi$ also as a character of $\mathbb{A}_{k}^{1}$ trivial on $J_{k}^{1}$. Note that $\tilde{\chi}$ is a character of $\mathbb{A}_{k}^{\times}$trivial on $J_{k}$. Both $L_{T}(s)$ and $\Xi_{x, T}\left(\omega_{s} \tilde{\chi}\right)$ are Dirichlet series in $s$ and, by Proposition 4.2 and Lemma 5.4, these series are absolutely convergent in the region $\operatorname{re}(s)>1$. Let us write

$$
L_{T}(s)=\sum_{m=1}^{\infty} l_{T, m}^{*} m^{-s}
$$

and

$$
\Xi_{x, T}\left(\omega_{s} \tilde{\chi}\right)=\sum_{m=1}^{\infty} a_{x, T, m}^{*}(\chi) m^{-s} .
$$

From Lemmas 5.3 and 5.4 and the usual rule for calculating the coefficients of a product of Dirichlet series, we see that

$$
l_{T, m}^{*}=\sum \prod_{v \notin T} l_{v, n_{v}}
$$

and

$$
a_{x, T, m}^{*}(\chi)=\sum \prod_{v \notin T} a_{i_{v}(x), v, n_{v}} \tilde{\chi}_{v}\left(\varpi_{v}\right)^{n_{v}}
$$

where the sum is over all factorizations $m=\prod_{v \notin T} q_{v}^{n_{v}}$. It follows that $a_{x, T, 1}^{*}(\chi)=1, l_{T, 1}^{*}=1$, $l_{T, m}^{*} \geqslant 0$ and $\left|a_{x, T, m}^{*}(\chi)\right| \leqslant l_{T, m}^{*}$ for all $x, T, m$ and $\chi$.

After these preliminaries, we introduce the Dirichlet series that are at the center of the our method. They are

$$
\xi_{\alpha, T}(s)=\sum_{x \in G_{k} \backslash X_{d}(\alpha)} o(x)^{-1} \Xi_{x, T}\left(\omega_{2 s}\right) \mathcal{N}\left(\Delta_{A_{x} / k}\right)^{-s}
$$

and

$$
\xi_{\alpha, T, \mathrm{e}}(s)=\frac{1}{h} \sum_{\chi} \sum_{x \in G_{k} \backslash X_{d}(\alpha)} o(x)^{-1} \chi\left(S\left(\mathcal{O}_{A_{x}}\right) \mathrm{C}^{-1}\right) \Xi_{x, T}\left(\omega_{2 s} \tilde{\chi}\right) \mathcal{N}\left(\Delta_{A_{x} / k}\right)^{-s}
$$




\section{A. C. Kable and D. J. Wright}

In the second equation, the outer sum is over all characters $\chi$ of $\mathrm{Cl}(k)$; we always understand such sums in this way. We must determine the analytic properties of these series. In order to do so, it is convenient to introduce the series

$$
\xi_{\beta, T}(s)=\sum_{x \in G_{k} \backslash X_{d}(\beta)} o(x)^{-1} \Xi_{x, T}\left(\omega_{2 s}\right) \mathcal{N}\left(\Delta_{A_{x} / k}\right)^{-s}
$$

and

$$
\xi_{\beta, T, \mathrm{e}}(s)=\frac{1}{h} \sum_{\chi} \sum_{x \in G_{k} \backslash X_{d}(\beta)} o(x)^{-1} \chi\left(S\left(\mathcal{O}_{A_{x}}\right) \mathrm{e}^{-1}\right) \Xi_{x, T}\left(\omega_{2 s} \tilde{\chi}\right) \mathcal{N}\left(\Delta_{A_{x} / k}\right)^{-s},
$$

which differ from $\xi_{\alpha, T}(s)$ and $\xi_{\alpha, T, \mathrm{e}}(s)$ only in that the sum includes fewer $x$. Note that we have

$$
\xi_{\alpha, T, \mathrm{e}}(s)=\sum_{\beta \mid T_{0}=\alpha} \xi_{\beta, T, \mathrm{e}}(s)
$$

and similarly with $\xi_{\alpha, T}(s)$. Thus, it suffices to determine the analytic properties of $\xi_{\beta, T}(s)$ and $\xi_{\beta, T, \mathrm{e}}(s)$.

The argument we are about to make is the same but for notation whether $d=2$ or $d=3$. However, for definiteness, we assume that $d=3$. Also we give the argument only for $\xi_{\beta, T, \mathrm{e}}(s)$, as the other is simpler. Consider the function

$$
I(s, \Phi)=\frac{1}{h} \sum_{\chi} \chi\left(\mathrm{e}^{-1}\right) Z_{3}(2 s, \chi, \Phi),
$$

where $\Phi \in \mathcal{S}_{0}\left(V_{\mathbb{A}}\right)$ is yet to be chosen. By Proposition 4.1, I( $\left.s, \Phi\right)$ is a holomorphic function in the region $\operatorname{re}(s)>1$, which has a meromorphic continuation to the region $\operatorname{re}(s)>5 / 6$, and whose only possible singularity in this region is a simple pole at $s=1$ with residue $\left(C_{3} / h\right) \Sigma_{3}(\Phi)$, where $C_{3}$ is a positive constant depending only on $k$. Let us suppose that $\operatorname{re}(s)>1$. By Proposition 4.3 , there is a positive constant $C_{0}$, depending only on $k$, such that

$$
I(s, \Phi)=\frac{C_{0}}{h} \sum_{\chi} \sum_{x \in G_{k} \backslash X_{3}} o(x)^{-1} \chi\left(S\left(\mathcal{O}_{A_{x}}\right) \mathcal{C}^{-1}\right) \Xi_{x}\left(\omega_{2 s} \tilde{\chi}, \Phi\right) \mathcal{N}\left(\Delta_{A_{x} / k}\right)^{-s} .
$$

We now make a particular choice of $\Phi$. For $v \in T \cap \mathfrak{M}_{\mathrm{f}}(k)$, let $\Phi_{1, v}$ denote the characteristic function of the set $U_{v} w_{\beta(v), v}$ and put

$$
\Phi=\bigotimes_{v \in \mathfrak{M}_{\infty}(k)} \Phi_{v} \otimes \bigotimes_{v \in T \cap \mathfrak{M}_{\mathrm{f}}(k)} \Phi_{1, v} \otimes \bigotimes_{v \notin T} \Phi_{0, v}
$$

where $\Phi_{v}$ for $v \in \mathfrak{M}_{\infty}(k)$ is chosen so that $\Phi_{v}$ is $U_{v}$-invariant, the support of $\Phi_{v}$ lies in $G_{k_{v}} w_{\beta(v), v}$, $Z_{\beta(v), v}\left(|\cdot|_{v}^{s}, \Phi_{v}\right)$ is entire and $Z_{\beta(v), v}\left(|\cdot|_{v}^{2}, \Phi_{v}\right) \neq 0$. According to Lemma 5.1 , these requirements at infinite places can be met. The restrictions on the supports of the various factors in $\Phi$ imply that $\Xi_{x}\left(\omega_{2 s} \tilde{\chi}, \Phi\right)=0$ unless $x \in X_{3}(\beta)$. Now suppose that $x \in X_{3}(\beta)$. If $v \in \mathfrak{M}_{\infty}(k)$ then $\tilde{\chi}_{v}$ is trivial and so

$$
\Xi_{x, v}\left(|\cdot|_{v}^{2 s} \tilde{\chi}_{v}, \Phi_{v}\right)=Z_{\beta(v), v}\left(|\cdot|_{v}^{2 s}, \Phi_{v}\right) .
$$

If $v \in T \cap \mathfrak{M}_{\mathrm{f}}(k)$ then, by the choice of $\Phi_{1, v}$ and the fact that $\tilde{\chi}_{v}$ is unramified,

$$
\Xi_{x, v}\left(|\cdot|_{v}^{2 s} \tilde{\chi}_{v}, \Phi_{1, v}\right)=Z_{\beta(v), v}\left(|\cdot|_{v}^{2 s} \tilde{\chi}_{v}, \Phi_{1, v}\right)
$$

is equal to the volume of the set $U_{v} H_{\beta(v), v}$, where $H_{\beta(v), v}$ denotes the isotropy group of $w_{\beta(v), v}$ in $G_{k_{v}}$, regardless of the values of $s$ and $\tilde{\chi}_{v}$. (Note that $H_{\beta(v), v}$ is a finite group. It is, in fact, always contained in $U_{v}$, but we do not require this.) In particular, we may write

$$
\Xi_{x, v}\left(|\cdot|_{v}^{2 s} \tilde{\chi}_{v}, \Phi_{1, v}\right)=Z_{\beta(v), v}\left(|\cdot|_{v}^{2}, \Phi_{1, v}\right)
$$




\section{UNIFORM DISTRIBUTION}

for all $v \in T \cap \mathfrak{M}_{\mathrm{f}}(k)$. On substituting these observations into (4), we obtain

$$
\xi_{\beta, T, \mathcal{e}}(s)=C_{0}^{-1} \prod_{v \in \mathfrak{M}_{\infty}(k)} Z_{\beta(v), v}\left(|\cdot|_{v}^{2 s}, \Phi_{v}\right)^{-1} \prod_{v \in T \cap \mathfrak{M}_{\mathrm{f}}(k)} Z_{\beta(v), v}\left(|\cdot|_{v}^{2}, \Phi_{1, v}\right)^{-1} I(s, \Phi) .
$$

To avoid needless repetition, we call a Dirichlet series standard if it converges absolutely in the region $\operatorname{re}(s)>1$ and extends meromorphically to the region $\operatorname{re}(s)>5 / 6$ with its only possible singularity a simple pole at $s=1$. We conclude from the previous equation that $\xi_{\beta, T, \mathrm{e}}(s)$ is standard. By using Lemma 5.2 , we see that the residue of $\xi_{\beta, T, \mathrm{e}}(s)$ at $s=1$ is

$$
\left(C_{3} C_{3}^{\prime} / h C_{0}\right) \prod_{v \in T} b_{\beta(v), v}^{-1} \prod_{v \notin T} \Sigma_{3, v}\left(\Phi_{0, v}\right) .
$$

By the choice of measures, $\Sigma_{3, v}\left(\Phi_{0, v}\right)=1$ for all $v \notin T$ and so this residue is actually $\left(C_{3} C_{3}^{\prime} / h C_{0}\right)$ $\prod_{v \in T} b_{\beta(v), v}^{-1}$. Equation (3) then implies that $\xi_{\alpha, T, \mathrm{e}}(s)$ is standard and that its residue at $s=1$ is $\left(C_{3} C_{3}^{\prime} / h C_{0}\right) \prod_{v \in T_{0}} b_{\alpha(v), v}^{-1} \prod_{v \in T \backslash T_{0}} E_{3, v}$. A simpler version of this argument shows that $\xi_{\alpha, T}(s)$ is standard and that its residue at $s=1$ is $\left(C_{3} C_{3}^{\prime} / C_{0}\right) \prod_{v \in T_{0}} b_{\alpha(v), v}^{-1} \prod_{v \in T \backslash T_{0}} E_{3, v}$. When $d=2$ we obtain the same conclusions and the residues of $\xi_{\alpha, T}(s)$ and $\xi_{\alpha, T, \mathrm{e}}(s)$ at $s=1$ in this case turn out to be $\left(C_{2} C_{2}^{\prime} / C_{0}\right) \prod_{v \in T_{0}} c_{\alpha(v), v}^{-1} \prod_{v \in T \backslash T_{0}} E_{2, v} \prod_{v \notin T}\left(1-q_{v}^{-2}\right)^{-1}$ and $1 / h$ times this quantity, respectively. The key fact, valid in both cases, is that

$$
\operatorname{res}_{s=1}\left(\xi_{\alpha, T, \mathfrak{e}}(s)\right)=\frac{1}{h} \operatorname{res}_{s=1}\left(\xi_{\alpha, T}(s)\right) .
$$

Now that (5) has been established, we may again treat the cases $d=2$ and $d=3$ simultaneously. Let us set

$$
\xi_{\alpha, T}(s)=\sum_{m=1}^{\infty} c_{\alpha, T, m} m^{-s}
$$

and

$$
\xi_{\alpha, T, \mathrm{e}}(s)=\sum_{m=1}^{\infty} c_{\alpha, T, m}(\mathrm{C}) m^{-s}
$$

Observe that

$$
c_{\alpha, T, m}=\sum_{\substack{x \in G_{k} \backslash X_{d}(\alpha) \\ n^{2} \mathcal{N}\left(\Delta_{A_{x} / k}\right)=m}} o(x)^{-1} a_{x, T, n}^{*}\left(\chi_{0}\right)
$$

and

$$
c_{\alpha, T, m}(\mathcal{C})=\frac{1}{h} \sum_{\substack{x \in G_{k} \backslash X_{d}(\alpha) \\ n^{2} \mathcal{N}\left(\Delta_{A_{x} / k}\right)=m}} \sum_{\chi} o(x)^{-1} \chi\left(S\left(\mathcal{O}_{A_{x}}\right) \mathcal{C}^{-1}\right) a_{x, T, n}^{*}(\chi) .
$$

From these equations and the properties of the $a_{x, T, n}^{*}(\chi)$ we obtain $c_{\alpha, T, m} \geqslant 0$ and $\left|c_{\alpha, T, m}(\mathcal{C})\right| \leqslant$ $c_{\alpha, T, m}$ for all $m \geqslant 1$. We now have all the hypotheses necessary to apply a standard Tauberian theorem ([Lan86, Theorem 1, §3, ch. 15], for example) to these standard Dirichlet series. As a consequence, we obtain

$$
\lim _{B \rightarrow \infty} B^{-1} \sum_{m \leqslant B}\left(c_{\alpha, T, m}(\mathcal{C})-\frac{1}{h} c_{\alpha, T, m}\right)=0
$$

for each $T$.

Let us write $c_{\alpha, T, m}=P_{m}+Q_{m}$ and $c_{\alpha, T, m}(\mathrm{C})=P_{m}(\mathrm{C})+Q_{m}(\mathrm{C})$, where $P_{m}$ and $P_{m}(\mathcal{C})$ are the parts of the above sums with $n=1$ and $Q_{m}$ and $Q_{m}(\mathcal{C})$ are the parts with $n \geqslant 2$. From the property $a_{x, T, 1}^{*}(\chi)=1$ for all $\chi$ and the orthogonality relation for the characters of $\mathrm{Cl}(k)$, we obtain 
the evaluations

$$
P_{m}=\sum_{\substack{x \in G_{k} \backslash X_{d}(\alpha) \\ \mathcal{N}\left(\Delta_{A_{x} / k}\right)=m}} o(x)^{-1}
$$

and

$$
P_{m}(\mathcal{C})=\sum_{\substack{x \in G_{k} \backslash X_{d}(\alpha, \mathcal{C}) \\ \mathcal{N}\left(\Delta_{A_{x} / k}\right)=m}} o(x)^{-1}
$$

We wish to estimate the terms $Q_{m}$ and $Q_{m}(\mathcal{C})$ on average. To this end, recall that both the set of quadratic fields and the set of cubic fields with prescribed splitting at a finite number of places are known to have a non-zero density. That is, there are non-zero constants $\mathcal{D}_{2}(\alpha)$ and $\mathcal{D}_{3}(\alpha)$ such that

$$
\lim _{B \rightarrow \infty} B^{-1} \sum_{x \in G_{k} \backslash X_{d}(\alpha, B)} o(x)^{-1}=\mathcal{D}_{d}(\alpha) .
$$

For $d=3$ this is the main result of [DW88]. For $d=2$ it is well known; it is stated as [DW88, Theorem 4.2], where possible references for a proof are given. In fact, a simpler version of the current proof would also serve to prove this fact. In any case, let us choose a constant $\mathcal{D}$ so large that

$$
\sum_{x \in G_{k} \backslash X_{d}(\alpha, B)} o(x)^{-1} \leqslant \mathcal{D} B
$$

for all $B>0$ (note that the left-hand side is 0 when $B<1$ ). From the fact that $\left|a_{x, T, n}^{*}(\chi)\right| \leqslant l_{T, n}^{*}$ we obtain

$$
\begin{aligned}
\left|B^{-1} \sum_{m \leqslant B} Q_{m}(\mathcal{C})\right| & \leqslant B^{-1} \sum_{\substack{x \in G_{k} \backslash X_{d}(\alpha) \\
n^{2} \mathcal{N}\left(\Delta_{A_{x} / k} \leqslant B, n \geqslant 2\right.}} o(x)^{-1} l_{T, n}^{*} \\
& =B^{-1} \sum_{n=2}^{\infty} l_{T, n}^{*} \sum_{x \in G_{k} \backslash X_{d}\left(\alpha, B n^{-2}\right)} o(x)^{-1} \\
& \leqslant \mathcal{D} \sum_{n=2}^{\infty} l_{T, n}^{*} n^{-2} \\
& =\mathcal{D}\left(L_{T}(2)-1\right)
\end{aligned}
$$

for $B>0$. A similar argument gives

$$
\left|B^{-1} \sum_{m \leqslant B} Q_{m}\right| \leqslant \mathcal{D}\left(L_{T}(2)-1\right)
$$

for $B>0$.

Let $\epsilon>0$. By Lemma 5.4, the product $\prod_{v \in \mathfrak{M}_{\mathrm{f}}(k)} L_{v}(2)$ is absolutely convergent and so it is possible to choose a finite set $T \supset T_{0}$ such that $\mathcal{D}\left(L_{T}(2)-1\right)<\epsilon / 4$. For this set $T$ we may, by (6), choose $B_{0}>0$ such that

$$
\left|B^{-1} \sum_{m \leqslant B}\left(c_{\alpha, T, m}(\mathcal{C})-\frac{1}{h} c_{\alpha, T, m}\right)\right|<\frac{\epsilon}{2}
$$

for all $B \geqslant B_{0}$. Together with the evaluations of $P_{m}$ and $P_{m}(\mathcal{C})$, these inequalities imply that

$$
\left|B^{-1} \sum_{x \in G_{k} \backslash X_{d}(\alpha, \mathcal{C}, B)} o(x)^{-1}-B^{-1} \frac{1}{h} \sum_{x \in G_{k} \backslash X_{d}(\alpha, B)} o(x)^{-1}\right|<\epsilon
$$

for all $B \geqslant B_{0}$. In combination with (7), this gives the required result. 


\section{UNIFORM DISTRIBUTION}

Corollary 5.6. Let $k$ be a number field, $h$ the class number of $k, \mathcal{C} \in \mathrm{Cl}(k)$ and $d \in\{2,3\}$. Choose a finite set $T_{0} \subset \mathfrak{M}(k)$, possibly empty, and for each $v \in T_{0}$ choose a separable $k_{v}$-algebra $\mathcal{A}_{v}$ of degree $d$ over $k_{v}$. For $B \geqslant 1$ let $F_{d}(B)$ denote the set of number fields $K$ such that $K \supset k, K / k$ has degree $d, \mathcal{N}\left(\Delta_{K / k}\right) \leqslant B$, and $k_{v} \otimes_{k} K \cong \mathcal{A}_{v}$ for every $v \in T_{0}$. If $d=3$ then we add the condition that $K / k$ be a non-normal extension. Let $F_{d}(\mathcal{C}, B) \subset F_{d}(B)$ be the set of those $K \in F_{d}(B)$ that also satisfy $S\left(\mathcal{O}_{K}\right)=\mathcal{C}$. Then we have

$$
\lim _{B \rightarrow \infty} \frac{\left|F_{d}(\mathcal{C}, B)\right|}{\left|F_{d}(B)\right|}=\frac{1}{h},
$$

where the vertical bars denote cardinality.

Proof. We first observe that the number of cyclic cubic extensions $K$ of $k$ with $\mathcal{N}\left(\Delta_{K / k}\right) \leqslant B$ is $o(B)$. It is a simple consequence of [Wri89, Theorem I.2] that this number is bounded by a constant times $B^{1 / 2} \log (B)$, which is more than enough. In light of (7), our claim follows immediately from Theorem 5.5 and the fact that $o(x)=\left|\operatorname{Aut}_{k}(K)\right|$ is 2 for all quadratic extensions and 1 for all non-normal cubic extensions of $k$. We have simply translated from the language of binary cubic forms to the language of number fields, using the Delone-Fadeev correspondence.

\section{ACKNowledgements}

The authors would like to thank Akihiko Yukie for useful comments on a first draft of this paper. The first-named author realized the significance of the invariant $S$ for the space of binary cubic forms whilst returning from a talk at the HAAR seminar at The Ohio State University. He would like to thank Eitan Sayag and Steve Rallis for stimulating discussions during his visit.

\section{REFERENCES}

Art65 E. Artin, Questions de base minimale dans la théorie des nombres algébriques, in Collected papers of Emil Artin (Addison-Wesley, Reading, MA, 1965), 229-321.

BBP04 K. Belabas, M. Bhargava and C. Pomerance, Error estimates for the Davenport-Heilbronn theorems, Preprint (2004).

Bou65 N. Bourbaki, Algèbre Commutative, Actualités Scientifiques et Industrielles, vol. 1314 (Hermann, Paris, 1965).

Car98 J. E. Carter, Module structure of integers in metacyclic extensions, Colloq. Math. 76 (1998), 191-199.

Coh00 H. Cohen, Advanced Topics in Computational Number Theory, Graduate Texts in Mathematics, vol. 193 (Springer, New York, 2000).

DW86 B. Datskovsky and D. J. Wright, The adelic zeta function associated to the space of binary cubic forms II: Local theory, J. reine angew. Math. 367 (1986), 27-75.

DW88 B. Datskovsky and D. J. Wright, Density of discriminants of cubic extensions, J. reine angew. Math. 386 (1988), 116-138.

DF64 B.N. Delone and D.K. Fadeev, Theory of Irrationalities of the Third Degree, Translated Mathematical Monographs, vol. 10 (American Mathematical Society, Providence, RI, 1964).

Fro60 A. Fröhlich, The discriminants of relative extensions and the existence of integral bases, Mathematika 7 (1960), 15-22.

FT91 A. Fröhlich and M. J. Taylor, Algebraic number theory, Cambridge Studies in Advanced Mathematics, vol. 27 (Cambridge University Press, Cambridge, 1991).

God02 M. Godin, Structure Galoisienne d'anneaux d'entiers, Thesis, L'Université de Valenciennes et du Hainaut Cambrésis, 2002.

GS03 M. Godin and B. Sodaïgui, Module structure of rings of integers in octahedral extensions, Acta Arith. 109 (2003), 321-327. 


\section{UNIFORM DISTRIBUTION}

KP00 C. Khare and D. Prasad, On the Steinitz module and capitulation of ideals, Nagoya Math. J. 160 (2000), 1-15.

Lan86 S. Lang, Algebraic Number Theory, Graduate Texts in Mathematics, vol. 110 (Springer, New York, 1986).

Rob01 D. P. Roberts, Density of cubic field discriminants, Math. Comp. 70 (2001), 1699-1705.

SS74 M. Sato and T. Shintani, On zeta functions associated with prehomogeneous vector spaces, Ann. of Math. (2) 100 (1974), 131-170.

Shi72 T. Shintani, On Dirichlet series whose coefficients are class-numbers of integral binary cubic forms, J. Math. Soc. Japan 24 (1972), 132-188.

Sov02 E. Soverchia, Steinitz classes of metacyclic extensions, J. London Math. Soc. (2) 66 (2002), 61-72.

Wei74 A. Weil, Basic Number Theory (Springer, Berlin, 1974).

Wri85 D. J. Wright, The adelic zeta function associated with the space of binary cubic forms I: Global theory, Math. Ann. 270 (1985), 503-534.

Wri89 D. J. Wright, Distribution of discriminants of abelian extensions, Proc. London Math. Soc. (3) 58 (1989), 17-50.

WY92 D. J. Wright and A. Yukie, Prehomogeneous vector spaces and field extensions, Invent. Math. 110 (1992), 283-314.

Yuk93 A. Yukie, Shintani zeta functions, London Mathematical Society Lecture Notes, vol. 183 (Cambridge University Press, Cambridge, 1993).

Yuk00 A. Yukie, Rational orbit decompositions of prehomogeneous vector spaces, Tohoku University Lecture Notes, June 2000, available at http://www.math.tohoku.ac.jp/ yukie/.

Yuk03 A. Yukie, On the density of cubic fields, Preprint (2003).

Anthony C. Kable akable@math.okstate.edu

Department of Mathematics, Oklahoma State University, Stillwater OK 74078, USA

David J. Wright wrightd@math.okstate.edu

Department of Mathematics, Oklahoma State University, Stillwater OK 74078, USA 